\title{
Physical activity prediction using fitness data: Challenges and issues
}

\author{
Nur Zarna Elya Zakariya, Marshima Mohd Rosli \\ Faculty of Computer \& Mathematical Sciences, Universiti Teknologi MARA, Malaysia
}

\begin{tabular}{l}
\hline \hline Article Info \\
\hline Article history: \\
Received Mar 8, 2020 \\
Revised May 24, 2020 \\
Accepted Jun 25, 2020 \\
\hline
\end{tabular}

\section{Keywords:}

Data personalization

Fitness data

Machine learning

Physical activity prediction

Wearable data

\begin{abstract}
In the new healthcare transformations, individuals are encourage to maintain healthy life based on their food diet and physical activity routine to avoid risk of serious disease. One of the recent healthcare technologies to support self health monitoring is wearable device that allow individual play active role on their own healthcare. However, there is still questions in terms of the accuracy of wearable data for recommending physical activity due to enormous fitness data generated by wearable devices. In this study, we conducted a literature review on machine learning techniques to predict suitable physical activities based on personal context and fitness data. We categorize and structure the research evidence that has been publish in the area of machine learning techniques for predicting physical activities using fitness data. We found 10 different models in behavior change technique (BCT) and we selected two suitable models which are fogg behavior model (FBM) and trans-theoretical behavior model (TTM) for predicting physical activity using fitness data. We proposed a conceptual framework which consists of personal fitness data, combination of TTM and FBM to predict the suitable physical activity based on personal context. This study will provide new insights in software development of healthcare technologies to support personalization of individuals in managing their own health.
\end{abstract}

This is an open access article under the CC BY-SA license.

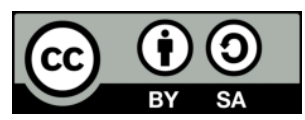

\section{Corresponding Author:}

Marshima Mohd Rosli,

Faculty of Computer \& Mathematical Sciences,

Universiti Teknologi MARA, 40450 Shah Alam, Selangor, Malaysia.

Email: marshima@fskm.uitm.edu.my

\section{INTRODUCTION}

Wearable technologies are devices and applications [1, 2] that popular nowadays for monitoring physical activities and prevention of diseases. The devices and applications are designed specifically to motivate individuals in monitoring their health, for example, diet tracking, weight control and physical activity tracking. The wearable devices synchronize with applications to generate daily fitness data such as number of steps, heart rate, calories burned, sleep tracking and distance of walking. In recent years, people are encourage to tracking their fitness data and maintain healthy diet to stay healthy and avoid risks of critical disease [3]. Some wearable devices and applications not only tracking fitness data but the applications also able to recommend a general physical activity to stay healthy. For example, adult aged 18-64 should do at least 150 minutes per week of moderate intensity aerobic physical activity in order to reduce the risk of chronic disease and depression [4]. Several initiatives have been explored in recent years to encourage physical activity with wearable technologies, particularly smartphones [5-7]. A study on adaptive physical activity promotion accessories had been conducted by Ledgers and McCaffrey reported that more than 50\% of people who bought wearable devices had stop using their wearable application after 6 months [8]. The 
researchers concluded that behavioral science methods are poorly implemented in the strategies to motivate people in using the wearable technologies, but the methods are effective and crucial to long-term engagement commitment [8]. Choe et al. studied how people reflect on self-monitoring fitness data and found that different wearable devices have generated different results for calculating the average of physical activity [9]. This indicates that there is still some concerns in terms of the accuracy of data for physical activity due to massive fitness data generated by the wearable devices [10]. One of the main concerns is the unique health personal context data that are captured in the wearable devices are not been considered for recommending the suitable physical activity $[11,12]$ such as age, weight and height. For example, a wearable application recommends an individual who is 70 years' old, weight $78 \mathrm{~kg}$ to walk 10.000 steps per day. This example indicates that the health personal context data are not taking into account for recommending the physical activities.

In this study, we review literature on machine learning techniques to predict suitable physical activities from fitness data. As we reviewed the literature, we observed 10 models in behavior change technique (BCT). We select two suitable models which are fogg behavior model (FBM) and trans-theoretical behavior model (TTM) with the eventual goal of predicting physical activity using personal context and fitness data. As first step towards this goal, we construct a framework called fitness personalization that consists of TTM and FBM. This framework describes the process to predict the suitable physical activity based on personal context and fitness data. The remaining of this paper is organized as follows: Section 2 describes the related work and section 3 describes the results of literature review. In section 4, we discuss the importance of findings from the literature review, followed by the proposed framework in section 5. Finally, we conclude and suggest future work in section 6.

\section{RELATED WORK}

\subsection{Techniques}

Developments in machine learning combined with emergence of low-cost processing or communication hardware technologies have led to ever-growing developments in computing and Internet-of-Things (IoT). There are two techniques that can be used for this study which are clustering and behavior change technique. Clustering process is for identifying contextual user groups and typical daily activity patterns within each contextual user group. BCT, the effective components of an intervention aimed at modifying existing behaviors or triggering new one. With innovations in online video, social networks, and metrics, the tools for creating persuasive products are becoming easier to use. As a result, across technology networks, more individuals and organizations will develop environments that they hope can affect the behaviors of people. In this study, we focus on BCT to predict the suitable physical activity from fitness data and personal context.

\subsection{Model}

In this section, we reviewed studies that discussed the BCTs based on fogg's behavior model, social cognitive theory (SCT), TTM, theory of planned behavior (TPB), I-change model, behavioral change wheel, activity theory, protection motivation theory, motivational interviewing, and health action process approach (HAPA) [6].

\subsubsection{Trans-theoretical}

Prochaska and DisClemente developed performance change model using TTM [13, 14] based on the assumptions that no single theory can account for the complexity of behavioral change because behavioral change is a multistage process that unfolds over time, stable and open to change, and specific processes and principles of changes that should be used at specific stages to maximize the effectiveness of behavior change. A study by [15], focused on to test people who are not under medical treatment but may have risk factors that can lead to chronic illness with pre-existing medical conditions. The main goal of the study is to increase their level of physical activity in their daily lives in order to meet the standard recommendations of health [4]. This study distinguishes four specific context categories which are time, location, activity and identity.

Time consists of day or night and weekdays or weekend. Location whether at gym or home. Details of activity consists of number of steps, burned calories, types of activity. Lastly, identity consists of gender, age, heart rate, blood pressure. Pre-contemplation, contemplation, preparation, action and maintenance are five different stages of an individual behavioral change in TTM [16]. Firstly, the individual does not intend to change his or her behavior in the near future in the pre-contemplation phase. Awareness rises in the stages of reflection and planning and the person takes first steps to change his or her actions. Phases of action and maintenance defines the phases where the new task is conducted and continued for a long time afterwards. This study used TTM to introduce a definition of context-conscious framework for encouraging physical activity. 


\subsubsection{Protection motivation theory}

Regarding public health, protection motivation theory (PMT) is important to understand how and why people act to protect their health $[17,18]$. One of the most commonly used public health methods for analyzing individual health habits is PMT [19], for instance, for assessing cancer prevention, healthy life style, and environmentally friendly activities [17, 18]. A study by [20], test the motivational interviewing effects of regular physical activity on the attitude and the intention of obese and overweight women guided by the PMT. In the study, 60 overweight and obese women attending health centers were selecting using convenience sampling. Weight was measured using a digital scale to the nearest $0.1 \mathrm{~kg}$, with the women dressed in light clothing. Height was measured using a height rod without shoes to the nearest millimeter. The waist circumference was measured in a standing position at the midpoint between the lower costal margin and the edge of the iliac crest. Body mass index (BMI) was the weight ratio (kg) to height square (m). The questionnaire has been designed and developed using seven PMT theory constructs which are sociodemographic characteristics, perceived sustainability, perceived severity, self-efficacy, perceived response efficacy, intention and attitude towards PA. Participants' academic status has been divided into four levels which are primary, intermediate, secondary and college. Present occupation has been listed as housewife, working, unemployed, retired or others (such as nongovernmental organization). Classification of marital status such as married or single. The results showed that anthropometric changes in the motivational interviewing group were more stable than those in the control group. In conclusion, findings suggest that PMT constructs may be successful in predicting the purpose of daily PA among women with overweight and obesity.

\subsubsection{Motivational interviewing}

A study by [21], examine the initial effectiveness of motivational assessments and cognitive behavioral therapies for long-term physical activity in adults with chronic conditions of health using motivational interviewing (MI). Information on age, gender, employment statues and health conditions was collected. Weight $(\mathrm{kg})$ has been measured in each center using calibrated scales. Clinical significance for weight was 5\% loss from baseline weight [22]. The BMI based in height and weight was calculates. Participants with stable conditions were randomized into a three-moth motivational interviewing and cognitive-behavioral group or usual care after completing a physical activity referral scheme. The results showed that the motivational interviewing and cognitive-behavioral group maintained kilocalorie expenditure at three and six months. Exercise barrier self-efficacy, physical and psychological physical activity experiences were increased at three months only.

\subsubsection{Social cognitive theory}

SCT claims that both culture and personal cognition affect human actions [23]. SCT describes human behavior as the triadic, complex, and reciprocal relationship between personal factors, actions and the environment [24]. A study by [25], evaluate the predictors of physical activity (PA) behavior among obese and overweight women in Borazian district us, south of Iran using SCT. Based on Pearson's correlation study, SCT concepts like self-efficacy, self-regulation, outcome preferences, and perceived family and friend social support are linked to actions of physical activity and energy expenditure. Information was collected including age, level of education, marital status, occupation and disease status. Education level are divided into four group which are elementary, high school, college, advanced degree. The seven day physical activity inventory questionnaire developed by Salis et al. was used to assess physical activity. The semi-structured interview form is used, in order to complete questionnaire. The question was asked during the interview about the PA that had taken place over past seven days based on duration, severity, type of activity. The results showed that physical activity were significantly related to employment status, education and marital status. Participants who were working, who were not married displayed a higher average daily energy expenditure by physical activity (TDEE) than other participants.

\subsubsection{Fogg behavior model}

A study by [26], discusses behavioral change management approaches aimed at promoting users' physical activity at various stages of the process of behavior change. There are three elements of fogg's model required in order to perform a target action [27] which are motivation, ability and trigger [28]. For example, healthy adults would put moderate to high on the exercise ability scale. Although there are obstacles such as lack of time, lack of money (to join a gym or take up a sport), or the weather, most people might find some time to do some kind of physical activity during their day if they wanted to [29]. More sophisticated features could add more data, such as heart rate and blood pressure to monitor changes in the measured values. While other factors such as gender and age have been shown to affect user acceptance. The approach helps to identify the health and fitness programs built for different types of user [26]. 


\subsubsection{Behavior change wheel}

Behavior change wheel (BCW) has become a popular choice for researchers seeking an intervention development guide [30]. The BCW structure was used to define behavior, identify roles for intervention and pick content and option for implementation [30]. Such variables help to understand "what needs to change." By placing behavioral determinants at its core, the BCW starts by identifying the factors that are most likely to cause behavioral change [31]. A study [32], to explore the attitudes of young women about health in the preconception era and the situational factors that affected everyday health behaviors. A researcher showed that young women in this urban African township understood the importance of a healthy diet and physical activity but lacked knowledge about the health and disease effects of overweight and obesity. Capability, opportunity and motivation are three rules of $\mathrm{BCW}$ in regulating behavior. Information including age, gender and ethnicity was collected. In addition, the model states that an individual must have the necessary skills and intention to perform a behavior, as well as no environmental constraints to prevent that behavior. The results indicated an obesogenic environment in which structural and social factors had a strong influence on the health choices of young women and restricted their ability to change behavior.

\subsubsection{I-change model}

A study by [33], perception precedes a person's motivation process and the impact on actions of pre-motivational factors are partly mediated by motivational factors by using I-change model (ICM) in order to understand motivational and behavioral change. Information were used are gender, age, height, weight and highest completed education level. Physical activity was measured in the international physical activity questionnaire (IPAQ). The IPAQ evaluated the frequency and the duration of walking, moderate-intensity activities and vigorous intensity activities. All three measurement points physical activity was measured and all analyzes were conducted for physical baseline activity. The results indicate that when tested separately, the associations of cognition, perception of risk and behavioral indications were fully mediated by motivational factors. The results suggest that pre-motivational factors are important to motivate, however, do not affect behavior directly. Table 1 shows that seven different models which are suitable to predict physical activity based on purpose and fitness data that had been used in their research.

Table 1. Difference between the types of models based on purpose and fitness data

\begin{tabular}{|c|c|c|}
\hline Model & Purpose & Fitness data \\
\hline Trans-theoretical & $\begin{array}{l}\text { To test people who are not under medical treatment but may } \\
\text { have risk factors that can lead to chronic illness with pre- } \\
\text { existing medical condition. }\end{array}$ & Time, location, activity, identity \\
\hline $\begin{array}{l}\text { Protection } \\
\text { motivation theory }\end{array}$ & $\begin{array}{l}\text { To test the motivational interviewing effects of regular } \\
\text { physical activity on the attitude and the intention of obese and } \\
\text { overweight women. }\end{array}$ & Weight, height, waist, BMI \\
\hline $\begin{array}{l}\text { Motivational } \\
\text { interviewing }\end{array}$ & $\begin{array}{l}\text { To examine the initial effectiveness of motivational } \\
\text { assessment of cognitive behavioral therapies for long-term } \\
\text { physical activity in adults with chronic conditions of health. }\end{array}$ & $\begin{array}{l}\text { Age, gender, employment status, } \\
\text { health condition, weight, height, } \\
\text { BMI }\end{array}$ \\
\hline $\begin{array}{l}\text { Social cognitive } \\
\text { theory }\end{array}$ & $\begin{array}{l}\text { To determine the predictors of physical activity (PA) behavior } \\
\text { in obese and overweight women in Borazian district, south of } \\
\text { Iran. }\end{array}$ & $\begin{array}{l}\text { Age, level of education, marital } \\
\text { status, occupation, disease status. }\end{array}$ \\
\hline $\begin{array}{l}\text { Fogg behavior } \\
\text { model }\end{array}$ & $\begin{array}{l}\text { To discuss behavioral change management approaches aimed } \\
\text { at promoting users' physical activity at various stages of the } \\
\text { process of behavior change. }\end{array}$ & $\begin{array}{l}\text { Age, gender, heart rate, blood } \\
\text { pressure }\end{array}$ \\
\hline $\begin{array}{l}\text { Behavior change } \\
\text { wheel }\end{array}$ & $\begin{array}{l}\text { To explore the attitudes of young women about health in the } \\
\text { preconception era and the situational factors that affected } \\
\text { everyday health behaviors. }\end{array}$ & Age, gender and ethnicity \\
\hline I-change model & $\begin{array}{l}\text { To understand motivational and behavioral change postulates } \\
\text { that a period of perception precedes a person's motivation. }\end{array}$ & $\begin{array}{l}\text { Gender, age, height, weight and } \\
\text { highest completed educational } \\
\text { level. }\end{array}$ \\
\hline
\end{tabular}

\section{RESULTS}

Figure 1 below shows one way to visualize the FBM. As the Figure 1 shows, the FBM has two axes. The vertical axis is built for motivation. A person with low motivation would register low on the vertical axis to perform the target behavior. High on the axis is highly motivated. The horizontal axis is for ability as shown in Figure 1. An individual with low ability to perform a target behavior would be identified on the left side of the axis. High ability is the right side. There is an arrow in Figure 1 that stretches diagonally across the plane from the bottom left to the top right. This arrow shows as an individual has improved motivation and ability, the more likely he or she is to perform the target activity. A behavior occurs when the user is sufficiently motivated, is capable of performing the behavior and is triggered to do so. 


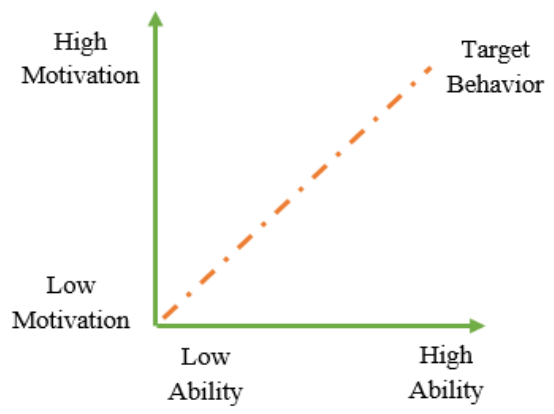

Figure 1. Fogg behavior model

The TTM defines an individual's behavioral change as a cycle in which he or she will move through five stages of change over time to transition a desired behavior. Individuals are believed not to change their behavior at once but they change it step by step or incrementally. Figure 2 shows that in order to change behavior, five stages of the TTM must be passed. Firstly, user in the pre-contemplation phase are inactive and unaware of the need for change. User step forward to the stage of contemplation and preparation stage when they became aware of the risk and plan to change their behavior. When user start a new behavior and keep it for a long time, user move to the action and last to the maintenance stage.

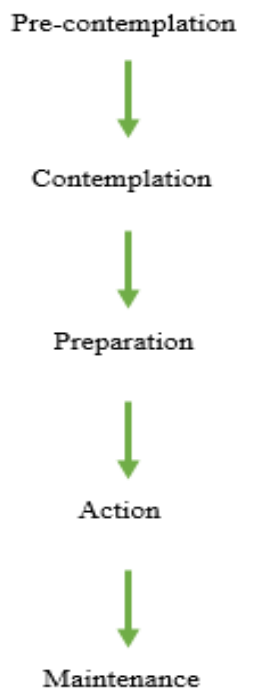

Figure 2. Five stage of TTM

Change processes consist of activities and strategies that help one progress in SOC and consist of two main stages. First, a cognitive process related to the thinking and feeling of individuals about unhealthy behavior. Second, behavior processes that lead to changes in unhealthy behavior.

a. Cognitive

- Motivational strategies (sparks)

Visualizing the benefits of improving physical activity such as improvements in the case of cardiovascular diseases, regular high blood pressure and diabetes, the current level of risk and the health benefits.

- Ability strategies (facilitators)

Showing, everyday life incentives for physical activity. Guidelines for the first step would minimize the brain cycles and instructions on how to integrate physical activity into the everyday routine.

- Triggers 
Monitoring of daily activity and physiological measurements such as body fat and weight. The ongoing engagement with the current situation gives the consumer's need for improvement and helps build awareness of risk.

b. Behavioral

- Motivational strategies (sparks)

Reward the user, even though small changes of the style of life towards physical activity. Rewards can be physical or in the form of digital points and milestones, such as financial rewards. Within social groups, being rewarded for physical activity can also be used to encourage other members to see their own success and ranking.

- $\quad$ Ability strategies (facilitators)

Knowing when the right time to do sports, where to do sports and how the right way to do sports and help to fit the right appliances for sports can improve people do physical activity. It through scheduling practices and prevents fallbacks such as missing training days.

- Triggers

Reminders that unhealthy behavior should be interrupted. It concentrated on everyday habits that can be modified quickly, but they are unified into everyday task. It requires specific calls for action to encourage the person to do something when the user mostly sufficient motivated but the change in behavior does not require considerable.

\section{DISCUSSION}

As mentioned by [34], a theoretical framework is lack in many research projects to predict physical activity. It may be better to use the combination of TTM and FBM to include behavior change theory in development software projects for predicting physical activity. TTM and FBM are two models that describe different aspects behavior change. The role in the behavior change process can be defined with the TTM, while the FBM discusses that psychological causes are discussed for behavior change. TTM originates from the field of psychology and is used in the context of behavioral change to distinguish user types into different stages. TTM consists of five different stages of positive behavioral change which are pre-contemplation, contemplation, preparation, action, maintenance. Next, FBM is used to incorporate techniques for operational intervention. This combination helps in the identification of the formed. Motivation, ability and effective trigger are three elements in order to perform a target behavior. In conclusion, the combination between FBM and trans-theoretical behavior model (TBM) helps to predict suitable physical activity designed for various types of user based on their fitness data.

\section{PROPOSED FRAMEWORK}

In this study, we construct a framework called fitness personalization. This framework consists of features, combination two models which are TBM and FBM. The two models are used to predict suitable physical activity based on fitness data and personal context collected from user. Figure 3 shows the fitness personalization framework.
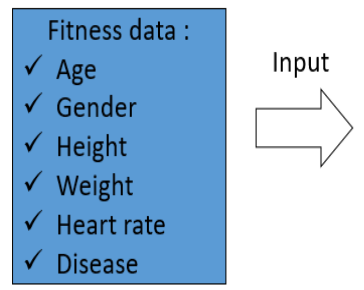

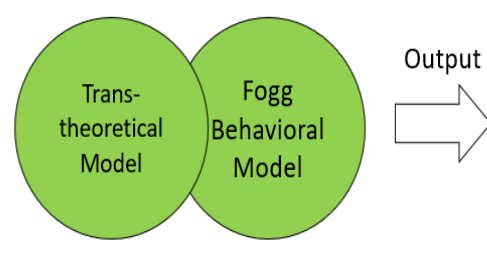

Figure 3. Framework

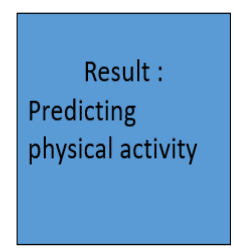

As described previously, the fitness data such as age, gender, heart rate and disease does not provide direct insight into the behavior of the user. Significant user knowledge needs to be extracted. Health analysis analyzes the health status of users and assesses whether current behavior has a positive or negative impact on wellbeing. This study will use combination model between trans-theoretical and fogg behavioral model (FBM). There are three principal factors for FBM which are motivation, ability and triggers. In brief, the model asserts that for a target behavior to happen, a person must have sufficient motivation, sufficient ability, 
and an effective trigger. All three must be present at the same instant for the behavior to occur. As researchers, the purpose of the FBM is to help think more clearly about behavior. Recent meta-analyzes suggested that TTM-based approaches are successful in encouraging physical activity and that processes of change and self-efficiency mechanisms are the TTM's most significant moderators in physical activity. In order to generate precise recommendations for physical activity, the guidelines from WHO are used [4]. For specific user based on the background details, guidelines on cardiovascular and muscular activity were selected. The synthesis of these strategies provides an overview of the user's current state of health as well as specific guidelines for changes in behavioral.

\section{CONCLUSION}

The purpose of this study is to review research papers about machine learning techniques in predicting physical activities from fitness data. Currently, there exist so many techniques for predictions in machine learning, but we observed 10 models that most appropriate for predicting physical activities using fitness data. We also identified few parameters and features that most appropriate to be used in predicting the suitable physical activity based on personal context. We found two models that considered important parameters in their process for predicting physical activities not only using fitness data but also using personal context data. We believe there is a need to develop an application for predicting suitable physical activities using fitness data and personal context data, and have taken a first step towards this goal by constructing the framework called fitness personalization, which consists of combination of TTM and FBM. The framework describes the process to predict suitable physical activity based on fitness data and personal context collected from the users. We are currently working on developing fitness personalization application for predicting physical activities that suitable based on features of individual. The fitness personalization application will predict based on personal context such as age, and fitness data are collected from wearable devices, such as number of walking steps and heart rate. It is hoped that this application will encourage people to continue maintaining their health with suitable physical activities according to their personal health context.

\section{ACKNOWLEDGMENTS}

The authors would like to thank the faculty of Computer and Mathematical Sciences, Universiti Teknologi Mara for their financial support to this research.

\section{REFERENCES}

[1] S. Abdullah, N. M. Noor, and M. Z. Ghazali, "Mobility recognition system for the visually impaired," 2014 IEEE 2nd International Symposium on Telecommunication Technologies (ISTT), Langkawi, pp. 362-367, 2014.

[2] F. H. Abdul Razak, K. Salleh, and N. H. Azmi, "Children's Technology: How Do Children Want It?," in International Visual Informatics Conference, pp. 275-284, 2013.

[4] S. Mutalib, A. Mohamed, S. Abdul-Rahman, and N. Mustafa, "Weighted frequent itemset of SNPs in genome wide studies," International Journal of Machine Learning and Computing, vol. 8, no. 4, August 2018.

[5] W. H. O. M. States and W. H. O. M. States, "Physical activity 23 Dec 2013," 2018.

[6] S. Consolvo, K. Everitt, I. Smith, and J. A. Landay, "Design requirements for technologies that encourage physical activity," Proceedings of the SIGCHI Conference on Human Factors in Computing Systems, vol. 1, pp. 457-466, April 2006.

[7] J. J. Lin, L. Mamykina, S. Lindtner, G. Delajoux, and H. B. Strub, "Fish'n'Steps: Encouraging physical activity with an interactive computer game," International Conference on Ubiquitous Computing, vol. 4206 LNCS, pp. 261-278, 2006.

[8] S. Consolvo et al., "Flowers or a robot army?: Encouraging awareness \& activity with personal, mobile displays," Proceedings of the 10th international conference on Ubiquitous computing, pp. 54-63, 2008.

[9] D. Ledger, "Inside Wearables - Part 2," Endeavour Partners, July 2014.

[10] S. Ghanvatkar, A. Kankanhalli, and V. Rajan, "User models for personalized physical activity interventions: Scoping review," JMIR mHealth and uHealth, vol. 7, no. 1, 2019.

[11] L. M. Feehan et al., "Accuracy of Fitbit Devices : Systematic Review and Narrative Syntheses of Quantitative Data Corresponding Author," JMIR mHealth and uHealth, vol. 6, no. 8, 2018.

[12] E. K. Choe, B. Lee, and H. Zhu, "Understanding Self-Reflection : How People Reflect on Personal Data through Visual Data Exploration," Proceedings of the 11th EAI International Conference on Pervasive Computing Technologies for Healthcare, pp. 173-182, May 2017.

[13] D. Huang et al., "Personal Visualization and Personal Visual Analytics", in IEEE Transactions on Visualization and Computer Graphics, vol. 21, no. 3, pp. 420-433, 1 March 2015.

[14] J. O. Prochaska and C. C. DiClemente, "Stages and processes of self-change of smoking: Toward an integrative model of change," Journal of Consulting and Clinical Psychology, vol. 51, no. 3, pp. 390-395, 1983. 
[15] A. Jerôme, C. Bortolon, M. Gourlan, and M. Carayol, "Matched or nonmatched interventions based on the transtheoretical model to promote physical activity . A meta-analysis of randomized controlled trials," Journal of Sport and Health Science, vol. 7, no. 1, pp. 50-57, 2018.

[16] A. Hamper, "A context aware mobile application for physical activity promotion," 2015 48th Hawaii International Conference on System Sciences, Kauai, HI, pp. 3197-3206, 2015.

[17] 1, C. Diclemente, and J. Norcross, "RR96-33 Baumol 1996.pdf," American Psychologist, vol. 47, no. 9, pp. 1102-1114, 1992.

[18] Floyd, D.L., Prentice-Dunn, S., \& Rogers, R.W, "A Meta-Analysis of research on protection motivation theory," Journal of Applied Social Psychology, vol. 30, no. 2, pp. 407-429, 2006.

[19] J. Wang, B. Liu-lastres, B. W. Ritchie, and D. J. Mills, “Annals of Tourism Research Travellers ' self-protections against health risks : An application of the full Protection Motivation Theory," Annals of Tourism Research, vol. 78, p. 102743, Sep 2019.

[20] P. Conner, M., \& Norman, "Predicting Health Behaviour: reseaarch and practice with social congnition model," Pergamon, vol. 24, no. 3, pp. 229-236, 1996.

[21] K. Mirkarimi et al., "Modifying attitude and intention toward regular physical activity using protection motivation theory: A randomized controlled trial," Eastern Mediterranean Health Journal La Revue de Santé de la Méditerranée orientale, vol. 23, no. 8, pp. 543-550, 2017.

[22] S. E. Scott, J. D. Breckon, and R. J. Copeland, "An integrated motivational interviewing and cognitive-behavioural intervention promoting physical activity maintenance for adults with chronic health conditions: A feasibility study," Chronic Illness, vol. 15, no. 4, pp. 276-292, 2019.

[23] D. L. Swift, N. M. Johannsen, C. J. Lavie, C. P. Earnest, and T. S. Church, "The role of exercise and physical activity in weight loss and maintenance," Progress in Cardiovascular Diseases, vol. 56, no. 4, pp. 441-447, 2014.

[24] Z. Tao, "Explaining virtual community user knowledge sharing based on social cognitive theory," 2008 4th International Conference on Wireless Communications, Networking and Mobile Computing, Dalian, pp. 1-4, 2008.

[25] S. Wang, K. Hung, and W. J. Huang, "Motivations for entrepreneurship in the tourism and hospitality sector: A social cognitive theory perspective," International Journal of Hospitality Management, vol. 78, pp. 78-88, April, 2019.

[26] L. Nami Nazari, H. Javazdzade, R. Tahmasebi, and M. Reisi, "Predictors of physical activity-related energy expenditure among overweight and obese middle-aged women in south of Iran: An application of social cognitive theory," Obesity Medicine, vol. 14, p. 100078, June 2019.

[27] A. Hamper, J. Wendt, C. Zagel, and F. Bodendorf, "Behavior change support for physical activity promotion: A theoretical view on mobile health and fitness applications," 2016 49th Hawaii International Conference on System Sciences (HICSS), Koloa, HI, pp. 3349-3358, 2016.

[28] B. Fogg, "A behavior model for persuasive design," Proceedings of the 4th International Conference on Persuasive Technology, no. 40, pp. 1-7, 2009.

[29] L. Ackermann, R. Mugge, and J. Schoormans, "Consumers' perspective on product care: An exploratory study of motivators, ability factors, and triggers," Journal of Cleaner Production, vol. 183, pp. 380-391, May 2018.

[30] E. M. Murtagh, A. T. Barnes, J. Mcmullen, and P. J. Morgan, "Mothers and teenage daughters walking to health: using the behaviour change wheel to develop an intervention to improve adolescent girls' physical activity," Public Health, vol. 158, pp. 37-46, 2018.

[31] C. Wilson and M. R. Marselle, "Energy Research \& Social Science Insights from psychology about the design and implementation of energy interventions using the Behaviour Change Wheel," Energy Research \& Social Science, vol. 19, pp. 177-191, Sep 2016.

[32] L. J. Ware et al., "Environmental, Social, and Structural Constraints for Health Behavior: Perceptions of Young Urban Black Women During the Preconception Period - A Healthy Life Trajectories Initiative," Journal of Nutrition Education and Behavior, vol. 51, no. 8, pp. 946-957, Sep 2019.

[33] S. Kasten, L. Van Osch, M. Candel, and H. De Vries, "The influence of pre-motivational factors on behavior via motivational factors: A test of the I-Change model," BMC Psychology, vol. 7, no. 1, pp. 1-12, 2019.

[34] J. Bort-Roig, N. D. Gilson, A. Puig-Ribera, R. S. Contreras, and S. G. Trost, "Measuring and influencing physical activity with smartphone technology: A systematic review," Sport. Medicine, vol. 44, no. 5, pp. 671-686, 2014. 